\title{
Theoretical study of B segregation in Mo(110)
}

\author{
Ceren Tayran*
}

Gazi University, Department of Physics, 06500 Ankara, Turkey, ORCID ID orcid.org/0000-0003-0185-0202

\author{
ARTICLE INFO \\ Article history: \\ Received 01 April 2018 \\ Revised form 17 October 2018 \\ Accepted 17 October 2018 \\ Available online 30 November 2018 \\ Research Article \\ DOI: 10.30728/boron.411591 \\ Keywords: \\ Density functional theory, \\ Atomic and electronic structure, \\ $\mathrm{Mo}(110)$ surface, \\ B atom, \\ Segregation
}

\begin{abstract}
Using density functional theory, we have calculated an atomic, electronic structures and energetics of the $\mathrm{B} / \mathrm{Mo}(110)$ surface and as well as from these calculations we have also studied B-segregation in the Mo substrate. The calculated segregation energy is negative which means that the non-segregated B-capped structure is disallow to be stable comparing the B atoms occupy the second-layer of Mo substrate. We have added new explanation for B segregation in Mo surface using the concept of bond numbers between Mo and $B$ atoms.
\end{abstract}

In the calculated electronic band structure of B segregation in the $\mathrm{Mo}(110)$ surface, we have determined a chemical bonding between Mo d-orbital and B p-orbitals which are clearly overlap. The other surface states are contributed individual orbital of Mo and B atoms.

\section{Introduction}

Transition metal surfaces such as ( $\mathrm{W}, \mathrm{Ta}, \mathrm{Mo}, \mathrm{Nb}$ ) have been studied by theoretically and experimentally with using various techniques. Their common properties are mainly to be cubic and closed packet structures. Regarding the adsorption of these metals with elemental atoms and molecules are also studied in order to enhance physical and chemical properties of their surfaces and interfaces. Among of them, the Mo(110) surface is also very attractive due to the industrial application. The stability and flatness of the Mo(110) surface allow adsorption and thin film growth [1-5]. Especially, some of recent study, C on the $\mathrm{Mo}(110)$ surface or with various molecules $[6,7], \mathrm{O}$ on $\mathrm{Mo}(110)$ [8,9], $\mathrm{H}$ on $\mathrm{Mo}(110)$ [10-13], $\mathrm{B}$ on $\mathrm{Mo}(110)$ [14] and $\mathrm{Sb}$ on $\mathrm{Mo}(110)$ [15] surface have been investigated their atomic and electronic properties. For example, one of the theoretical study for $\mathrm{O}$ adsorption on the Mo(110) surface is studied by Zhou et al. [9] where $O$ placed on-surface and subsurface sites. They found that $O$ prefers to stay hollow site and the work-function increases with rising coverage at this site. They also showed that subsurface adsorption sites are less stable than on-surface sites.

Another interesting element is boron which is a unique element of the group 13 of the periodic table. It is known that due to the electron deficient, bonding nature for boron increases reactiveness with chemical compounds with very different elements. In general review of heavy transition metal borides have been broadly investigated to understand the potential ultra-incompressible and hard materials [16-33].
Molybdenum borides, due to their mechanical properties [27-32], can be easily synthesized under ambient pressure conditions. Thus, they are highly preferred in industrial applications on this view. It is necessary to say that it is very difficult to determine the crystal structure of $\mathrm{Mo}_{x} \mathrm{~B}_{y}$ experimentally by $\mathrm{x}$-ray diffraction (XRD) patterns since multicenter bonds and $\mathrm{sp}, \mathrm{sp}^{2}$, $\mathrm{sp}^{3}$ hybridization of these bonds [34-36]. The crystal structure of molybdenum borides is a problem that remains incomplete. In studies, crystal structures of molybdenum borides are predicted by ab initio method [28-33]. One of these studies, the $\mathrm{Mo}_{2} \mathrm{~B}(14 / \mathrm{m})$ structure is found to be mechanically and dynamically stable while $\mathrm{Mo}_{2} \mathrm{~B}\left(\mathrm{Al}_{2} \mathrm{Cu}\right.$-type $\left.(14 / \mathrm{mcm})\right)$ is unstable [33]. It indicates ultra-incompressible behavior since it has strong three-dimensional covalent Mo-B bonds. These above alloy systems can be different in physically and chemically on surface. In theoretical aspect, these can be analyzed by using simple physical arguments such as surface segregation with $B$ and Mo. In experimental aspect, the study by Magkoev and coworker [14] using Auger electron spectroscopy (AES), x-ray photoelectron spectroscopy (XPS), electron energy loss spectroscopy (EELS) and work function measurements, they have clearly indicated the penetration of B atoms into Mo substrate. Their another important finding was about bonds between $B$ and Mo atoms and related state of B-Mo bond.

As far as we know that there is no any theoretical study regarding B on Mo surface. In order to clarify physical and chemical behavior of $B$ in the Mo(110) surface, we have studied $B$ segregation in the Mo(110) surface by using density functional theory. We have 
found in energetic point of view that the $B$ segregation in the $\mathrm{Mo}(110)$ surface is favorable considering $B$ atom topmost layer of Mo.

\section{Computational methods}

All the calculations performed with Vienna Ab-initio simulation package (VASP) [37-38]. Perdew, Burke and Ernzerhof (PBE) functional [39] was represented for exchange-correlation. The ion-electron interaction is described by the Projector-augmented-wave (PAW) method [40] potentials with a cut off energy of $600 \mathrm{eV}$ for plane-wave basis expansion. A set of $20 \times 20 \times 1$ Monkhorst-Pack [41] k-points were adopted for the sample of Brillouin zone (BZ). Geometry optimization was processed until, the total energy convergence was set to be $1 \times 10^{-6}$ eV/atom and Hellmann-Feynman force forces on the atoms less than $0.01 \mathrm{eV} / \AA$. The optimized lattice parameter is $3.151 \AA$ which is good agreement with experimental value of $\sim 3.15 \AA$ [42-44] and the theoretical calculated value of $3.16 \AA$ and $3.153 \AA[8,9]$.

The clean Mo(110) surface was modeled by slab geometry including nine layers of Mo with $30 \AA$ vacuum thickness (making supercell). After force acting test on Mo, the vacuum region and the thickness of slab are found to be enough that the surface does not affect from the deeper layers of Mo. In this symmetric slab with $1 \times 1$ unit cell, all the atoms have been allowed to relax into their minimum energy position except middle three Mo layers. When our calculations included spinpolarization, there are any difference in energies and lattice parameter. For this reason, our results do not include spin-polarization calculation.

\section{Results and discussion}

We have first explored the clean Mo(110) surface with nine layers. The optimized atomic structure of clean $\mathrm{Mo}(110)$ and its related electronic band structures are given in Figure 1.a and Figure 2.a, respectively.

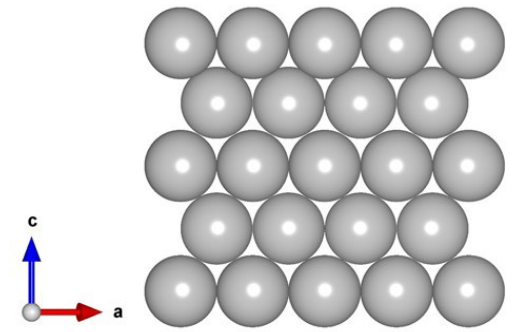

(a)

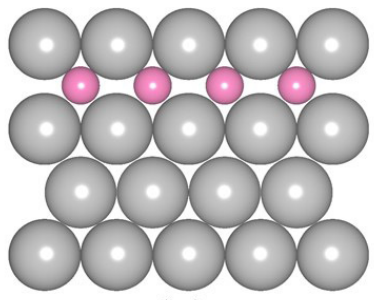

(c)
We have determined a total of seven surface states $\left(S_{1}-S_{7}\right)$. The clean Mo surface shows the metallic character as expected. For comparison reason, Rot et al. [45] studied Li/W(110) and Li/Mo(110) surfaces, the electronic band structures of Mo bulk projected and clean Mo(110) surface were given in their work. Their experimental data from AES are shown as solid circle in Figure 1.a. As seen from Figure 1.a, our results are excellent agreement with their study. The states, $S_{1}-S_{3}$, are below the Fermi level and mainly related to Mo-Mo atoms interaction with some contribution d-orbital (4 lobes are in the z-direction) of the Mo atoms as seen in Figure 3.a. The states, $S_{4}-S_{7}$, are mainly contributed from Mo surface atoms and some degree of contribution from bulk Mo atoms. Their orbital characters are drawn between the point of $\bar{\Gamma}-\overline{\mathrm{H}}$ and shown in Figure 3.b.

After these, we have focused B segregation in the $\mathrm{Mo}(110)$ surface. It means that Mo atom replaced by a $B$ atom, allowing segregation of $B$ atoms into the first, second, and third layers. The atomic structures are presented in Figures 1.b-c. In order to understand the segregation characterization of the $B$ atom in $\mathrm{Mo}(110)$ surface, the segregation energies $E_{\text {seg }}$ have been calculated as following equation;

$E_{\text {seg }}=E_{M o(110)(B, n t h-l a y e r)}-E_{M o(110)(B, 3 r d-l a y e r)}$

where $E_{M o(110)(B, n t h \text {-layer })}$ is the total energy of B is located in $\mathrm{n}^{\text {th }}(\mathrm{n}=1$ or 2$) \operatorname{Mo}(110)$ surface and $E_{M o(110)(B, 3 r d-l a y e r)}$ indicates the total energy of $\mathrm{B}$ is located in 3rd atomic layer. When this equation gives a negative value, $B$ atom segregates through the Mo(110) surface.

The calculated surface segregation energies using Eq.1 are given in Table 1. According to our calculations, the segregation energy is found to be $-0.45 \mathrm{eV}$ where $B$ is located in 2 nd layer. This result shows a

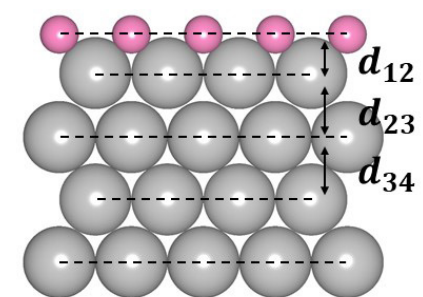

(b)

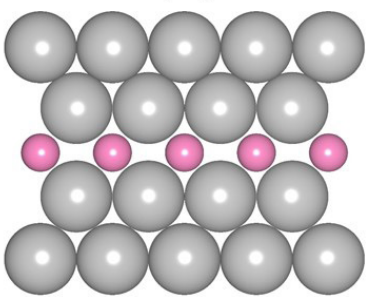

(d)

Figure 1. The atomic structure of a) clean, b) B atom at first layer c) B atom at second layer and d) B atom at third layer. Gray and pink balls indicate $\mathrm{Mo}$ and $\mathrm{B}$ atoms, respectively. $d_{12}$ represents vertical distance between layers. Only the five top layers are schematically shown. 


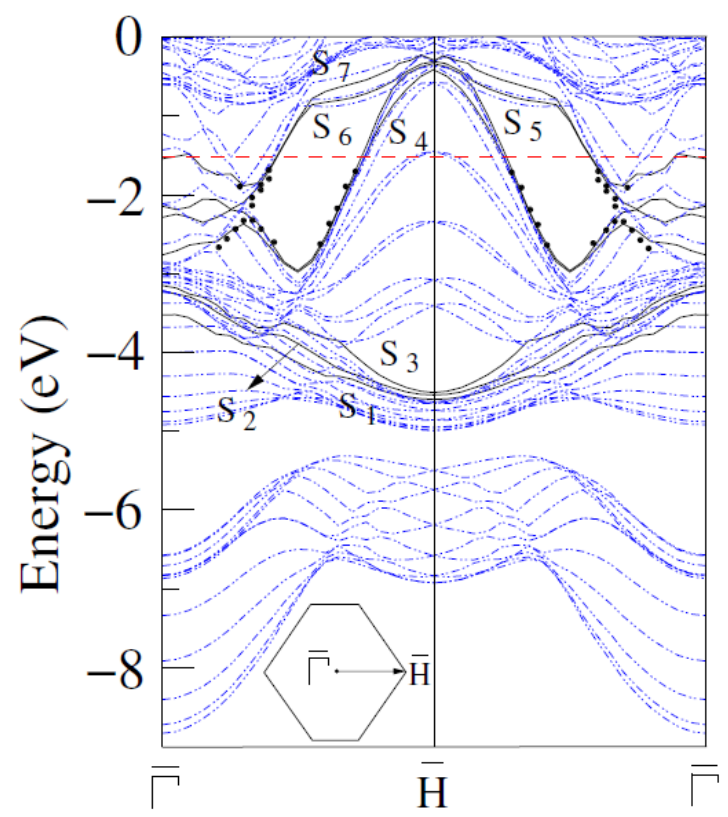

(a)

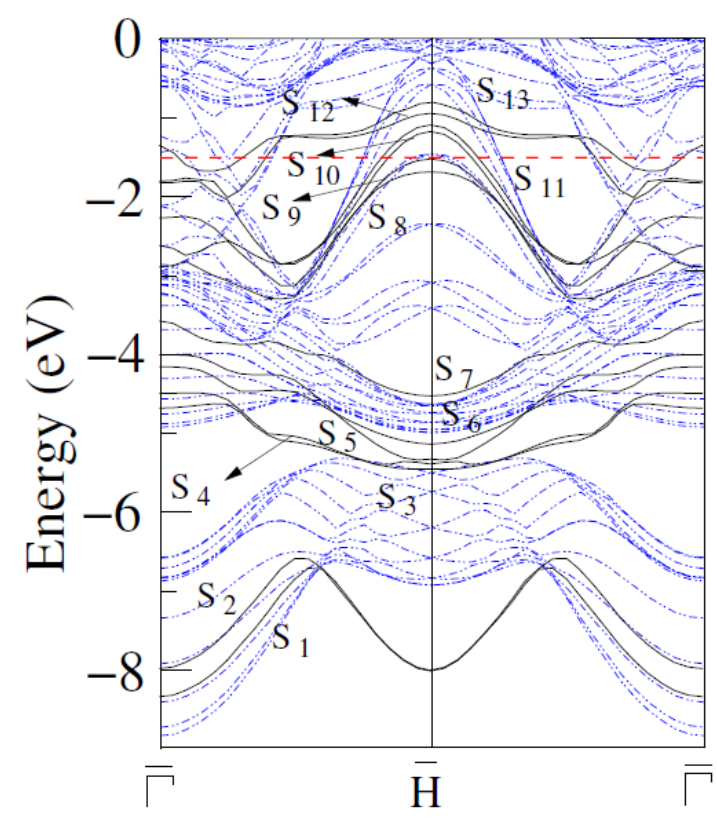

(b)

Figure 2. Electronic band structures of a) clean and b) B atom at second layer. The blue dashed lines, black solid lines and red dashed line represent electronic band structure of Mo bulk projected, surface states and Fermi Level, respectively. The solid circles are taken from AES measurement [14]. Also, the Surface Brilliouin Zone (SBZ) is shown schematically.

clear segregation than the others. When $B$ is located in 1st layer, the segregation energy is found to be +1.45 $\mathrm{eV}$. This positive result represents that $\mathrm{B}$ does not prefer to stay at the first layer.

In previous study, Yu et al. [46] investigated Mo segregation in MoNi(111) system using DFT, and found Mo atom prefer to stay in second layer. In addition, another work of them considered some transition metals segregation in $\mathrm{Ni}(111)$ surface such as $\mathrm{W}, \mathrm{Mo}, \mathrm{V}$, $\mathrm{Fe}, \mathrm{Cr}$ [47]. These elements prefer to occupy a site in the second layer. They also analyzed energy lowering in segregation using two rules; (i) avoiding the higher surface energy of $B$ and (ii) by locating at the second layer, the system can largely release the elastic energy due to the atomic size mismatch. In addition to that, we could add another rule about segregation. The number of bonds between $B$ and $\mathrm{Mo}$ is increased from 2 to 4 when the $B$ atoms are segregated to the dipper layers. This could also dominate factor for segregation preferentially into dipper layers.

Furthermore, to pave the way to future experimental studies, we have calculated key parameter such as vertical distance $d_{i j}$ from the Mo to Mo(or B) atoms between the $i^{t h}$ and $j^{\text {th }}$ layer. The calculated values are listed in Table 1 and labels are shown in Figures 1.b-d. It is interesting to say that when the $B$ atom at the second layer, the perpendicular distance between $\mathrm{B}$ and $\mathrm{Mo}$ is elongated to $\approx \% 12$, comparing with $\mathrm{B}$ at the first layer case. This result actually promotes the rules explained above.

As calculated segregation energy point of view, the calculated electronic band structure corresponding to
B segregation in the 2nd layer is presented in Figure 3.b. Due to overlap of Fermi level with surface states, it shows metallic in character. We have determined a total of thirteen surface states as labelled $S_{1}-S_{13}$ Their charge density plots are depicted in Figures 4.a-f. Figure 4.a. represents the states $S_{1}$ and $S_{2}$ and mainly related to B atoms. The states $S_{3}$ and $S_{5}$ are mainly the similar orbital characters and comes from topmost Mo atoms. The interesting orbital character is depicted in Figure 4.c. for $S_{4}$ and $S_{6}$. These states clearly indicate the interaction of $B$ atoms with surrounding Mo atoms. However, only topmost surface Mo atoms and B atoms interaction can be contributed the states of $S_{8}$ and $S_{9}$. The complex interesting orbital character can be seen in Figure 4.d which are mainly d-orbital of topmost Mo atoms and some contribution comes from p-orbital $\mathrm{B}$ atoms. The states $S_{7}$ is entirely bulk region. The states $S_{10}$ and $S_{11}$ are related to only topmost Mo atoms. These states are similar to states $S_{4}-S_{5}$ for the clean case, i.e. without B atom case. Interesting d-orbital character can be seen in Figure 4.f which makes the surface states of $S_{12}$ and $S_{13}$.

In Magkoev et. al. [14] studies, they have found clearly changes in the electronic structure due to the $B$ penetration into Mo(110) using concept of surface plasmon mode. Furthermore, in their study, they have also indicated possible Mo-B chemical bonding formations. These two clear indicators can be comparable with present study as discussed above. We find explicitly that the $B$ atoms can be found energetically favorable when the $B$ atom is underneath of topmost surface of $\mathrm{Mo}(110)$. This phenomena can be described as segregation of $B$ in $\mathrm{Mo}(110)$. For the calculated electronic band structure for the case $\mathrm{B} / \mathrm{Mo}(110)$, the chemical 


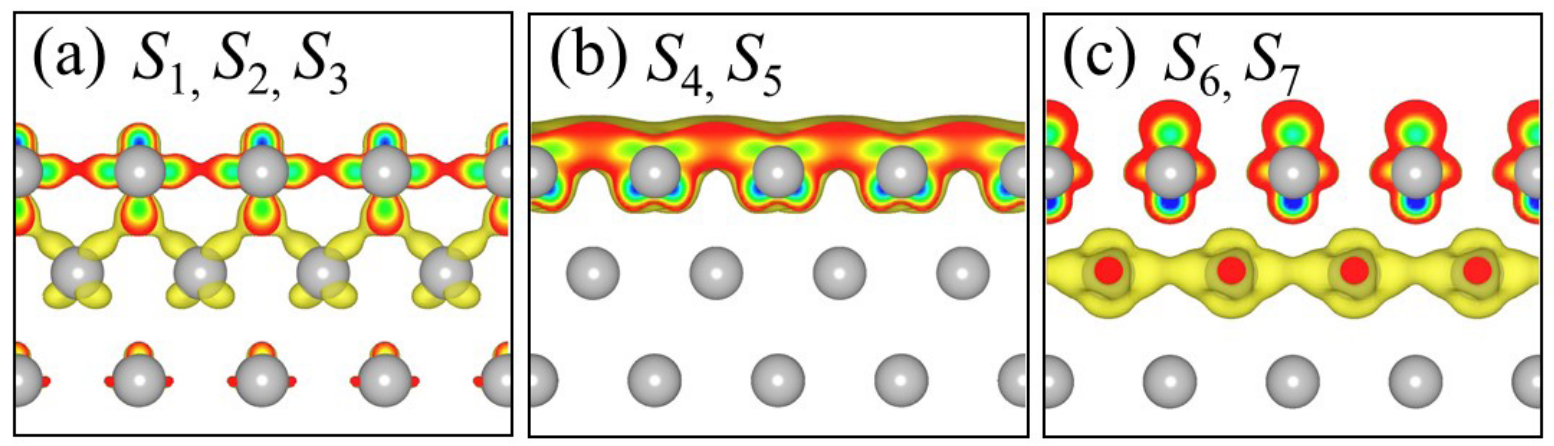

Figure 3. The electronic charge density plots of the individual states for the clean $\mathrm{Mo}(110)$ surface. On the plane cutting vertically through the Mo atoms (a) $S_{1}-S_{3}$ at the $\overline{\mathrm{H}}$ point, (b) $S_{4}$ and $S_{5}$ between the point of $\bar{\Gamma}-\overline{\mathrm{H}}$ and (c) $S_{6}$ and $S_{7}$ between the point of $\bar{\Gamma}-\overline{\mathrm{H}}$.

Table 1. The relative energies (in eV) for B segregation at the layers of $\mathrm{Mo}(110)$ and the calculated structural parameter (in $\AA$ ).

\begin{tabular}{ccccc}
\hline Position of the $\mathbf{B}$ & $\boldsymbol{\Delta} \mathbf{E}(\mathbf{e V})$ & $\boldsymbol{d}_{\mathbf{1 2}}(\AA)$ & $\boldsymbol{d}_{\mathbf{2 3}}(\AA)$ & $\boldsymbol{d}_{\mathbf{3 4}}(\AA)$ \\
\hline First layer & +1.18 & 1.37 & 2.27 & 2.22 \\
Second layer & -0.45 & 1.48 & 1.55 & 2.26 \\
Third layer & 0.00 & 2.17 & 1.59 & 1.56 \\
\hline
\end{tabular}
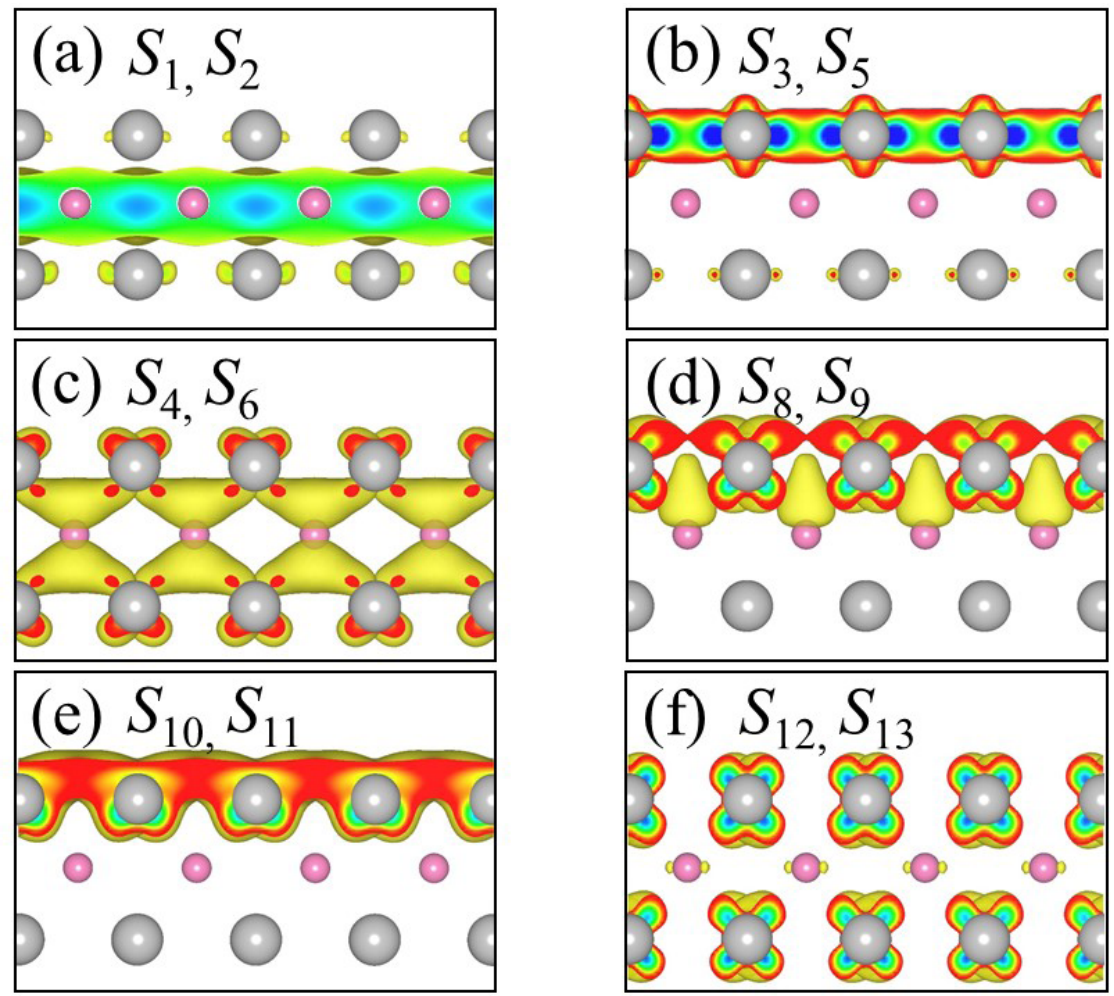

Figure 4. The electronic charge density plots of the individual states for B segregation at the second layer of Mo(110). On the plane cutting vertically through the Mo-B atoms a) $S_{1}$ and $S_{2}$ at the $\overline{\mathrm{H}}$ point, b) $S_{3}$ and $S_{5}$ at the $\overline{\mathrm{H}}$, c) $S_{4}$ and $S_{6}$ between the point of $\overline{\mathrm{H}}$, d) $S_{8}$ and $S_{9}$ between the point of $\overline{\mathrm{H}}, \mathrm{e}) S_{10}, S_{11}$ and f) $S_{12}$ and $S_{13}$. between the point of $\bar{\Gamma}-\overline{\mathrm{H}}$.

bonds between Mo-B can be seen as surface states which are fully or partially overlap of the orbitals come from Mo- and B atoms. The only overlap can be seen in the states $S_{4}$ and $S_{6}$ (q.v. Figure 4.c) can be comparable with the experimental measurements for chemical bonding between Mo- and B. The d-orbital of Mo is overlap with $\mathrm{p}$-orbital of $\mathrm{B}$.

\section{Conclusion}

Segregation behavior of $B$ atoms in $M o(110)$ surface was investigated by means of density functional theory. The results are indicated that the $\mathrm{B}$ atom prefers to stay underneath of the topmost Mo surface as energetically. We have also calculated the electronic band structures and determined the surface related states. It is interesting to say that the chemical bondings have been calculated for only in two states due the overlap between Mo d-orbital and B p-orbitals. The other surface states are contributed individual orbital of Mo and $\mathrm{B}$ atoms.

\section{Acknowledgments}

This work also was financially supported by the Ministry of Development of TR under Project No: 
2016K121220. The author thanks Prof. Dr. Mehmet ÇAKMAK for valuable contribution.

\section{References}

[1] Chen J. G., Menning C. A., Zellner M. B., Monolayer bimetallic surfaces: Experimental and theoretical studies of trends in electronic and chemical properties, Surf. Sci. Rep., 63, 201-254, 2008.

[2] Horn K., Scheffler M., Electronic Structure, Handbook of Surface Science Amsterdam: North-Holland, 2000.

[3] Somorjai G. A., Introduction to Surface Chemistry and Catalysis, Wiley New York, 1994.

[4] Bonzel P., Bradshaw A. M., Ertl G., Physics and Chemistry of Alkali Metal Adsorption Elsevier Amsterdam, 1989.

[5] Bol'shov L. A, Napartovich A. I., Naumovets A. G., Fedorus A., G., Submonolayer films on the surface of metals, Sov. Phys. Usp. 20, 432-451, 1977.

[6] Gleichweit C., Neiss C., Maisel S., Bauer U., Späth F., Höfert O., Vollnhals F., Drost M., Marbach H., Görling A., Steinrück H-P., Papp C., Comparative study of the carbide-modified surfaces $\mathrm{C} / \mathrm{Mo}(110)$ and $\mathrm{C} / \mathrm{Mo}(100)$ using high-resolution $\mathrm{x}$-ray photoelectron spectroscopy, Phys. Rev. B, 92, 0141142015.

[7] Bauer U., Gleichweit C., Höfert O., Späth F., Gotterbarm K., Steinrück H-P., Papp C., Reactivity studies of ethylene, benzene and cyclohexane on carbide-modified $\mathrm{Mo}(110)$ using high resolution X-ray photoelectron spectroscopy, Surf. Sci., 000 1-9 article in press, 2018.

[8] Petrova N. V., Yakovkin I. N., Density-functional and Monte Carlo study of $\mathrm{O} / \mathrm{Mo}(110)$ : Structures and desorption, Phys. Rev. B, 76, 205401, 2007.

[9] Zhou Y. G., Zu X. T., Nie J. L., Gao F., Adsorption of $\mathrm{O}$ on $\mathrm{Mo}(110)$ surface from first-principles calculation, Eur. Phys. J., B, 67, 27-34, 2009.

[10] Arnold M., Sologuby S., Frie W., Hammer L. Heinz K., Hydrogen-induced buckling of $\mathrm{Mo}(110)$ at submonolayer coverage, J. Phys.: Condens. Matter. , 9, 6481649, 1997

[11] Wang T., Tian X., Yang Y., Li Y-W., Wang J., Bellera M., Jiao H., Structures of seven molybdenum surfaces and their coverage dependent hydrogen adsorption, Chem. Phys., 18, 6005-6012, 2016.

[12] Altman M., Chung J. W., Estrup P. J., Kosterlitz J. M., Prybyla J., Sahu D., Ying S. C., Phase transformations of the $\mathrm{H} / \mathrm{W}(110)$ and $\mathrm{H} / \mathrm{Mo}(110)$ surfaces, J. Vac. Sci. Technol., A, 5, 10451987.

[13] Kohler B., Ruggerone P., Wilke S. and Scheffler M., Frustrated H-Induced Instability of Mo (110), Phys. Rev. Lett., 74, 1387, 1995.

[14] Magkoev T. T., Turievl A. M., Tsidaeva N. I., Panteleev D. G., Vladimirov G. G., Rump G. A., Adsorption of boron on a $\mathrm{Mo}(110)$ surface, J. Phys. Condens. Matter., 20, 485007, 2008.

[15] Proskurin D., Nikolaychik A., Koval I.F. and Yakavkin I. N., Electronic structure of Sb monolayers on the Mo(110) surface, Physica Stat. Solidi B, 243, 584, 2006.
[16] Cumberland R. W., Weinberger M. B., Gilman J. J., Clark S. M., Tolbert S. H., Kaner R. B., Osmium Diboride, An Ultra-Incompressible, Hard Material, J. Am. Chem. Soc., 127, 7264-7265, 2005.

[17] Chung H. Y., Weinberger M. B., Levine J. B., Kavner A., Yang J. M., Tolbert S. H., Kaner R. B., Synthesis of ultra-incompressible superhard rhenium diboride at ambient pressure, Sci., 316, 436, 2007.

[18] Qin J. Q., He D. W., Wang J. H., Fang L. M., Lei L., Li Y. J., Hu J., Kou Z. L., Bi Y., Is Rhenium Diboride a Superhard Material?, Adv. Mater. 20, 4780-4783, 2008.

[19] Dubrovinskaia N., Dubrovinsky L. Solozhenko V. L., Comment on "Synthesis of Ultra-Incompressible Superhard Rhenium Diboride at Ambient Pressure", Sci., 318, 1550c, 2007.

[20] Li Q., Zhou D., Zheng W., Ma Y., Chen C., Global Structural Optimization of Tungsten Borides, Phys. Rev. Lett. 110, 136403, 2013.

[21] Mohammadi R., Lech A. T., Xie M., Weaver B. E., Yeung M. T., Tolbert S. H., Kaner R. B., Tungsten tetraboride, an inexpensive superhard material, Proc. Natl. Acad. Sci. U. S. A. 108, 10958-10962, 2011.

[22] Gu Q., Krauss F., Steurer W., Transition Metal Borides: Superhard versus Ultra-incompressible, Adv. Mater. 20, 3620-3626, 2008.

[23] Niu H., Wang J., Chen X.-Q., Li D., Li Y., Lazar P., Podloucky R., Kolmogorov A. N., Structure, bonding, and possible superhardness of $\mathrm{CrB}_{4}$, Phys. Rev. B, 85, 144116, 2012.

[24] Li Q., Wang H., Ma Y. M., Predicting new superhard phases, J. Superhard Mater. 32, 192-204, 2010.

[25] Tian Y., Xu B., Zhao Z., Microscopic theory of hardness and design of novel superhard crystals, Int. J. Refract. Met. Hard Mater. 33, 93-106, 2012.

[26] Chung H. Y., Yang J. M., Tolbert S. H., Kanerb R. B., Anisotropic mechanical properties of ultra-incompressible, hard osmium diboride, J. Mater. Res. 23, 17971801, 2008.

[27] Tao Q., Zhao X., Chen Y., Li J., Li Q., Ma Y., Li J., Cui T., Zhu P., Wang X., Enhanced Vickers hardness by quasi-3D boron network in $\mathrm{MoB}_{2}$, RSC Adv. 3, 1831718322, 2013.

[28] Zhang M., Wang H., Wang H., Cui T., Ma Y., Structural Modifications and Mechanical Properties of Molybdenum Borides from First Principles, J. Phys. Chem. C, 114, 6722-6725, 2010.

[29] Okada S., Atoda T., Higashi I., Takahashi Y., Preparation of single crystals of $\mathrm{MoB}_{2}$ by the aluminium-flux technique and some of their properties, J. Mater. Sci., 22, 2993-2999, 1987.

[30] Kiessling R., The crystal structures of molybdenum and tungsten borides, Acta Chem. Scand. 1, 893-916, 1947.

[31] Kudaka K., lizumi K., Sasaki T., Okada S., Mechanochemical synthesis of $\mathrm{MoB}_{2}$ and $\mathrm{Mo}_{2} \mathrm{~B}_{5}$, J. Alloys Compd. 315, 104-107, 2001.

[32] Frotscher M., Klein W., Bauer J., Fang C. M., Halet J. 
F., Senyshyn A., Baehtz C., Albert B. Z, $M_{2} B_{5}$ or $M_{2} B_{4}$ ? $A$ Reinvestigation of the Mo/B and W/B System, $Z$. Anorg. Allg. Chem., 633, 2626-2630, 2007.

[33] Zhou D., Wang J., Cui Q., Li Q., Crystal structure and physical properties of $\mathrm{Mo}_{2} \mathrm{~B}$ : First-principle calculations, J. App. Phys., 115, 113504, 2014.

[34] OganovA. R., Solozhenko V. L., Boron: A hunt for superhard polymorphs, J. Superhard Mater. 31, 285, 2009.

[35] Oganov A. R., Chen J., Gatti C., Ma Y., Glass C. W., Liu Z., Yu T., Kurakevych O. O., Solozhenko V. L., Ionic high-pressure form of elemental boron, Nature 457, 863-867, 2009.

[36] Zhou W., Sun H., Chen C., Soft Bond-Deformation Paths in Superhard Y-Boron, Phys. Rev. Lett. 105, 215503, 2010.

[37] Efficiency of ab-initio total energy calculations for metals and semiconductors using a plane-wave basis set, Comp. Mat. Sci., 6, 15-50, 1996.

[38] Kresse G., Furtmüller J., Efficient iterative schemes for ab initio total-energy calculations using a plane-wave basis set, Phys. Rev. B, 54, 11169, 1996.

[39] Perdew J. P., Burke K., Ernzerhof M., Generalized Gradient Approximation Made Simple, Phys. Rev. Lett. 77, 3865, 1996.
[40] Blochl P., Projector augmented-wave method, Phys. Rev. B, 50, 17953, 1994.

[41] Monkhorst H., Pack J., Special points for Brillouinzone integrations, Phys. Rev. B, 13, 5188, 1976.

[42] Katahara K. W., Manghnani M. H., Fisher E. S., Pressure derivatives of the elastic moduli of BCC Ti-V-Cr, Nb-Mo and Ta-W alloys, J. Phys. F 9, 773, 1979.

[43] Mattheiss L. F. and Hamann D.R., Linear augmentedplane-wave calculation of the structural properties of bulk Cr, Mo, and W, Phys. Rev. B, 33, 823, 1986.

[44] Zunger A., Cohen M. L., Self-consistent pseudopotential calculation of the bulk properties of Mo and W, Phys. Rev. B, 19, 568 ,1979.

[45] Rotenberg E., Chung J. W., Kevan S. D., Spin-Orbit Coupling Induced Surface Band Splitting in Li/W(110) and Li/Mo(110), Phys. Rev. Lett. 82, 4066-4069, 1999.

[46] Yu Y., Xiao W., Wang J., Wang L., Correction: FirstPrinciples Study of Mo Segregation in MoNi(111): Effects of Chemisorbed Atomic Oxygen, Materials 9, 352, 2016.

[47] Yu Y., Xiao W., Wang J.and Wang L., Understanding the surface segregation behavior of transition metals on $\mathrm{Ni}(111)$ : a first-principles study, Chem. Phys. 18, 26616-26622, 2016. 